\title{
Theoretical prerequisites for improving the body low loader granular fertilizer spreader
}

\author{
Shwarz Anatoly ${ }^{1, *}$, Korotkov Ilya ${ }^{1}$, Shwarz Sergey ${ }^{2}$ and Vetrov Ivan ${ }^{1}$ \\ ${ }^{1}$ Federal State Budgetary Educational Institution of Higher Education "Kursk State Agricultural Academy named after I. I. Ivanov ", 70, \\ Karl Marx street, Kursk, Russia \\ ${ }^{2}$ LLC "Meridian", 11, Ufimtsev street, Kursk, Russia
}

\begin{abstract}
It is known that the use of granular forms of fertilizers is a universal solution for plant growing enterprises using organic, mineral, organomineral fertilizers in their activities. For crop farming enterprises, the application of granular fertilizers is the most convenient way to enrich the soil with nutrients. For the surface main application and top dressing, spreaders of different designs are used, distributing fertilizers in a continuous layer with different indicators of uniformity. At present, the development of machines of various designs is underway to operate applying solid fertilizers. The main tasks of research by various authors in the field of mechanization of the distribution of fertilizers over the surface of the field are to reduce the uneven distribution of fertilizers across the width, increase the sowing width, as well as reduce the changeover time for the fertilization operation. The article presents studies related to the improvement of the design of the working bodies of the machine for applying granular fertilizers, installed on the horizontal axis of rotation. To achieve the goal of increasing the working width of the unit without reducing the quality of the sifting, an option was proposed to supplement the design of a low-frame body spreader for solid fertilizers.
\end{abstract}

\section{Introduction}

At present, for the application of granular and powder-like fertilizers in agricultural production, fertilizer distributors are mounted or trailed with different designs of working bodies: centrifugal with blades on vertical or horizontal axes of rotation, pendulum, or rod type [1]. In Russian and foreign production of fertilizer machines, developments are underway to improve all the above designs. The main tasks of research in this area are to reduce the uneven distribution of fertilizers across the width, increase the productivity of machines, which is closely associated with an increase in the sowing width, as well as reducing the time spent on the shift for the fertilizer application $[2,3]$.

Machines with centrifugal working bodies located on horizontal axes of rotation have not found wide application in mass production, however, several researchers consider it possible and expedient to improve and use such devices for sieving fertilizers [4]. One of the options for further research in the direction of modernizing machines is to install a second rotor on the fertilizer distributor for sifting fertilizers in the opposite direction. However, when using such a design, it will be necessary to supplement the design for seeding the strip directly under the machine.

\footnotetext{
*Corresponding author: shwarz@internet.ru
}

\section{Problem Statement}

Theoretical and experimental studies on the distribution of fertilizers by a spreader with blades on a horizontal axis of rotation have shown the effectiveness of using a cover and a deflector to achieve an increase in the spreading width. The use of such a constructive solution made it possible to increase the sieving distance of fertilizer particles to $18 \mathrm{~m}$ while maintaining the permissible indicators of uniformity of distribution (Figure 1).

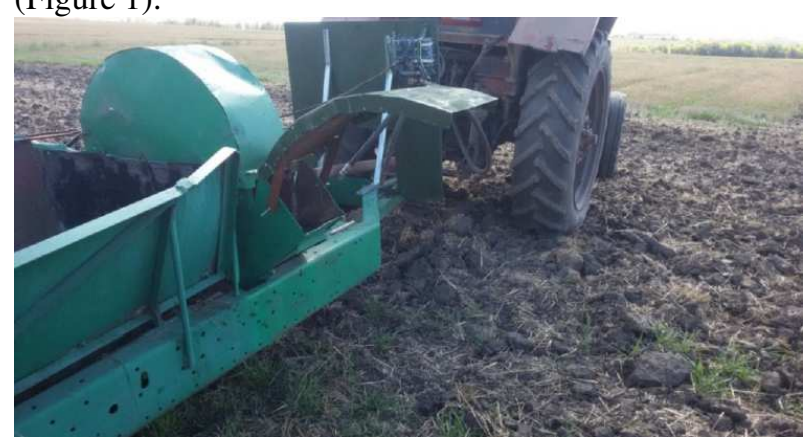

Fig. 1. Deflector with a radius of $980 \mathrm{~mm}$ when working with a rotor with a radius of $460 \mathrm{~mm}$.

Further studies on the distribution of fertilizers across the width show that when two vertical rotors with covers are used on a body spreader, an unseeded strip under the 
machine with a width of about $7 \mathrm{~m}$ will remain. If this deficiency can be compensated, the total distribution width will increase significantly. In order to install rotors with a diameter of $700 \mathrm{~mm}$, each sieving width will be $14+14=28 \mathrm{~m}$ with an unseeded strip between them about $7 \mathrm{~m}$, the sifting width of powdered superphosphate with one rotor with a diameter of $920 \mathrm{~mm}$ will be only $18 \mathrm{~m}$ [5] ( Figure 2).

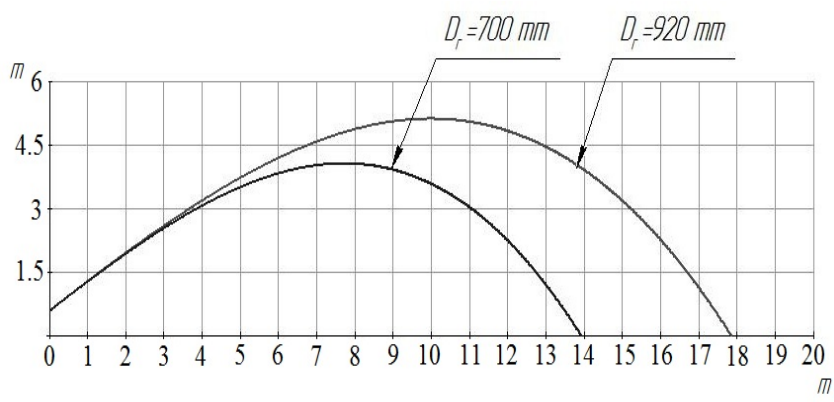

Fig. 2. Flight range of powder superphosphate particles with rotors with diameters of $700 \mathrm{~mm}$ and $920 \mathrm{~mm}$.

Consequently, for the productive operation of a fertilizer spreader of such a design, it becomes necessary to install an additional working body capable of distributing fertilizers over a width of at least 7 meters.

\section{Research Questions}

To achieve the task of uniform distribution of fertilizers over a width of up to 35 meters, it is planned to install an additional spreading device between two rotors with horizontal axes of rotation (Figure 3).
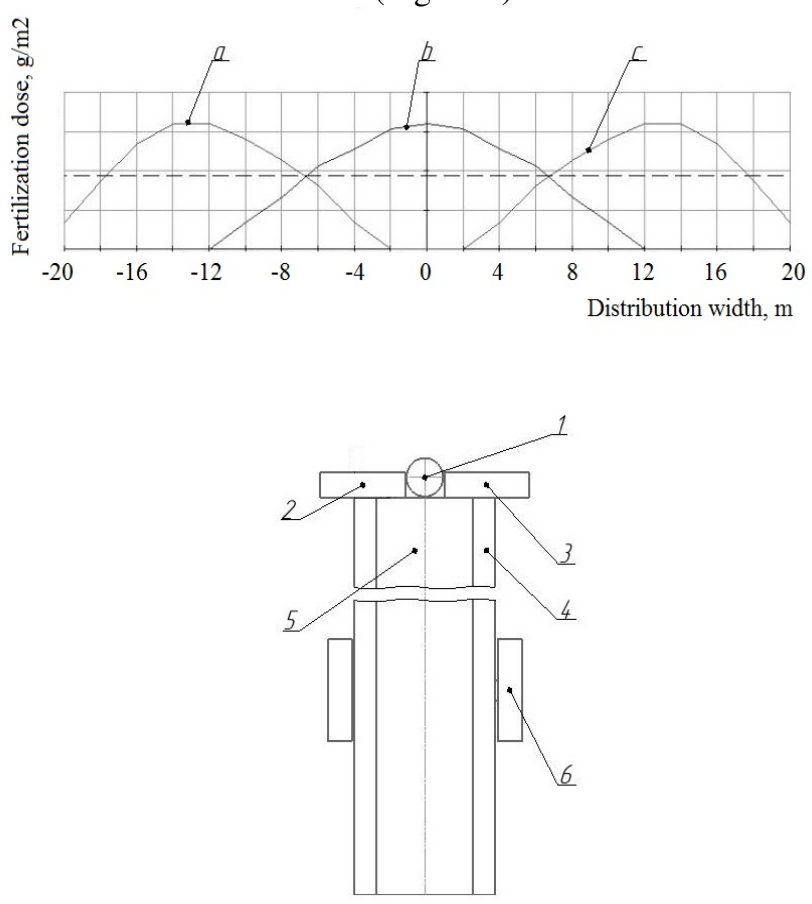

Fig. 3. Scheme of a granular fertilizer spreader: a - fertilizer application rate with the left rotor; $b$ - the dose of fertilization by the additional spreading device; $\mathrm{c}$ - the dose of fertilization by the right rotor; 1 - spreading disc; 2 - left rotor; 3 - right rotor; 4 - spreader body; 5- belt conveyor; 6 - chassis.
A distribution plate on a vertical axis of rotation can serve as such a device. However, the design of the experimental spreader of the Kursk State Agricultural Academy assumes several limitations for solving such a problem (Figure 4).

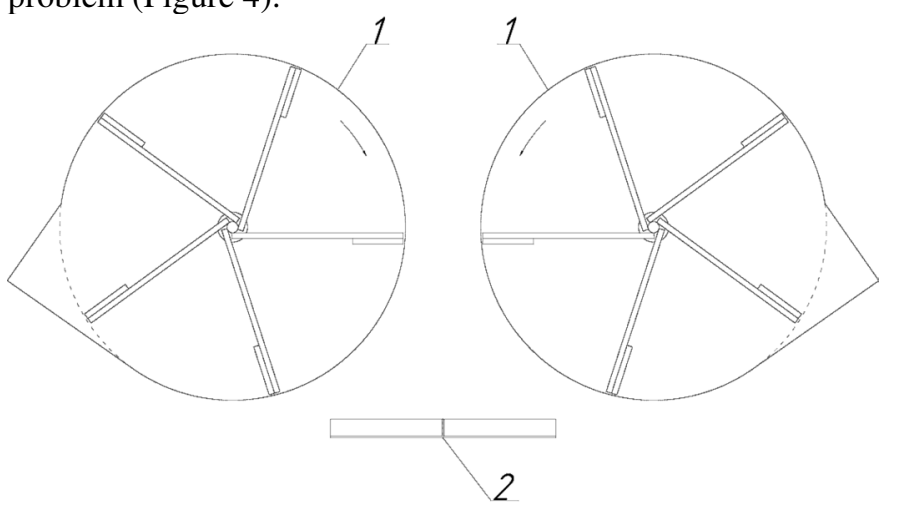

Fig. 4. Layout of the working bodies of the low-bed solid fertilizer spreader: 1 - rotors; 2 - spreading disc.

One of the advantages of a fertilizer distributor designed by the Kursk State Agricultural Academy is its low-frame design, which allows loading the machine directly from the body of a dump truck. This loading option allows you to significantly save shift time, reduce the number of personnel serving the fertilizer application process, and reduce the cost of fuel and lubricants (Figure 5).

\section{loading position}

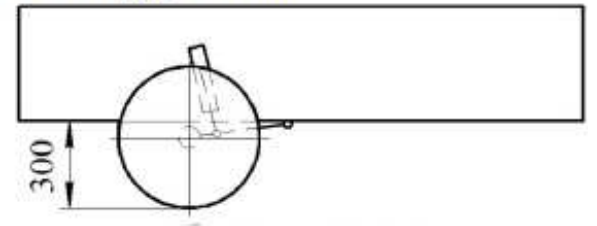

\section{working position}

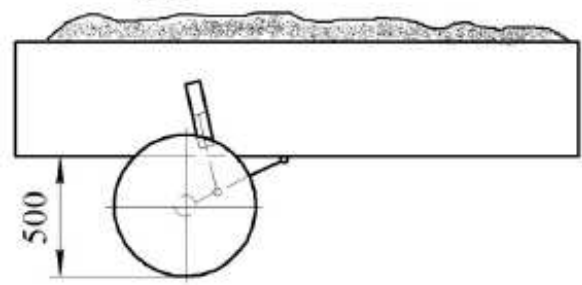

Fig. 5. The position of the body of the low loader fertilizer spreader.

The position of the body of the low-frame fertilizer distributor in this case is regulated by two hydraulic cylinders connected to the crankshafts. In cases where the movement of the fertilizer spreader is carried out across the slope, which is an agrotechnical requirement when working in rough terrain, one of the hydraulic cylinders is also used to stabilize the spatial position of the body in the horizontal plane. 
For the same purpose, a pendulum-type sensor with a valve-spool distributor P-75V2 is used (Figure 6). The stabilization device works as follows: when the body is tilted laterally, the pendulum, while maintaining its vertical position, displaces the corresponding spool of the hydraulic distributor and turns the hydraulic system into operation. The hydraulic cylinder of the lower downhill wheel, rotates the crank rack of the half-axle with the support wheel until the pendulum moves the spool of the hydraulic distributor to the neutral position.

In a situation where the spreader maintains a horizontal position when working on slopes, a uniform supply of material to the loading window of the distributor rotor is achieved and, as a result, a uniform distribution of fertilizers on the slope. When adding a second rotor and a plate to the spreader design, the pendulum stabilizer will help maintain the supply of the required fertilizer doses to each of the three spreading devices, preventing clogging of the working bodies and maintaining the specified dose and width of the sieving.

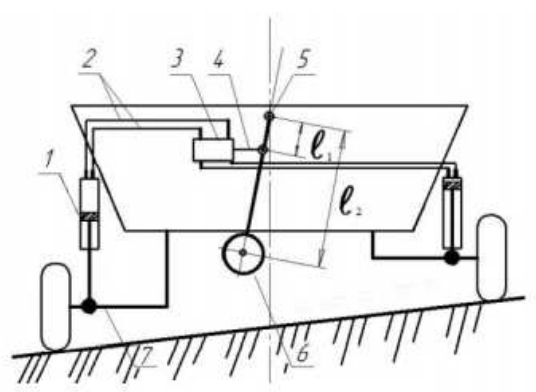

a

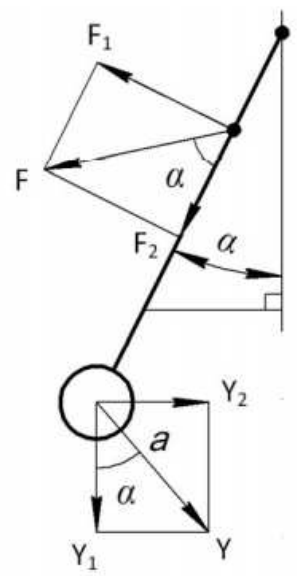

b

Fig. 6. Device (a) and force diagram of the stabilization system (b)of the low-frame spreader: 1 - hydraulic cylinder; 2 pipelines; 3 - hydraulic distributor; 4 - hydraulic spreader spool; 5 - pendulum type lever; 6-load; 7-crankshaft.
However, this design makes it difficult to install a centrifugal dispersing device. The maximum height at which the distributor plate can be installed provided the low-frame structure is preserved, is $0.5 \mathrm{~m}$. However, this is consistent with the opinion of Y. I. Yakimov, in whose work it is said that to reduce the influence of the slope angle and wind speed, it is advisable to use spreaders use flat discs with a height of not more than $0.5 \mathrm{~m}$ above the soil [6].

The second limiting factor for the installation of the spreading disc is the width of the low bed spreader body. Thus, the experimental spreader of the Kursk State Agricultural Academy with a working body on the horizontal axis of rotation is equipped with a prismatic body with the upper base width of $1160 \mathrm{~mm}$, the lower one $-800 \mathrm{~mm}$, which allows the material to be fed into the end of the rotor with a diameter of $920 \mathrm{~mm}$. It is possible to install two rotors with a diameter of $700 \mathrm{~mm}$ each (Figure 5).

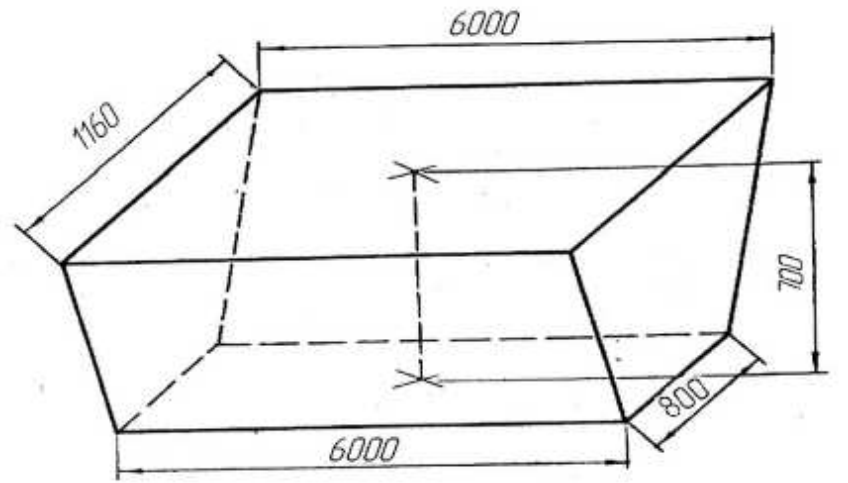

Fig. 5. Low bed spreader body shape

For the effectiveness of the study, it is necessary to determine the minimum size of the scattering plate, its diameter, as well as the length of the blades.

\subsection{Purpose of the Study}

The main task of this work is to determine the minimum dimensions of the centrifugal spreading device for the required operating parameters of the experimental granular fertilizer spreader of the Kursk State Agricultural Academy, considering the above-mentioned height restrictions.

\subsection{Research Methods}

The article uses different methods such as analysis, synthesis, generalization, and mathematical modeling.

\section{Findings}

The working bodies of the disc design of most fertilizer spreaders rotate at a frequency of $540 \ldots 980$ min-1. In particular, the rotational speed of the Rostselmash Grach 900 spreader discs is 730 min-1 [7], for Rauch MDS 55 
this figure corresponds to $540 \mathrm{~min}-1$ [8], AMAZONE ZA-M 1500 discs rotate at $720 \mathrm{~min}-1$ [9], and the nominal rotational speed of the RDU-1.5 disks is 980 min-1 [10].

The peripheral speed of the plate is calculated by the following formula

$$
V=\frac{\pi d n}{60}
$$

where $\mathrm{d}$ is the diameter of the plate, $\mathrm{m}$;

$$
\mathrm{n} \text { - rotation frequency, } \min ^{-1} \text {. }
$$

Consequently, the peripheral speed of rotation of the plate is in direct proportion to its diameter and speed.

To reduce the cost of the design, we take the simplest variant of the arrangement of the spreading disc blades radially from the center of the circle [11]. For the same purpose, we assume the use of a flat disc (the value of the taper angle will be $0^{\circ}$ ).

Thus, the equation of motion of the ejected particle will be as follows

$$
y=x \cdot \operatorname{tg} \alpha-\frac{g}{V_{0}^{2} \cdot \cos \alpha},
$$

where $\mathrm{x}, \mathrm{y}$ are the current coordinates, and $\mathrm{x}$ is directed horizontally towards the exit of the particle, $y$ is directed vertically upward;

$\alpha$ - disc taper angle;

$V_{0}$ - particle initial velocity.

The initial velocity of the particle $V_{0}$ is equal to the peripheral velocity of the edge of the spreading disc vane. In addition to the initial velocity $V_{0}$ and the taper angle $\alpha$, the flight range of particles 1 is influenced by the height of the disk above the field. Consequently, at a taper angle $\alpha=0$, a plate height $\mathrm{h}=0.5 \mathrm{~m}$, the equation takes the form

$$
\frac{9.81 x^{2}}{V_{0}^{2}}=0.5
$$

To achieve a sieve width of $7 \mathrm{~m}$, the flight range of the particles must be at least $3.5 \mathrm{~m}$. The peripheral speed will be

$$
V=\sqrt{\frac{9.81 x^{2}}{0.5}}=\sqrt{\frac{9.81 \cdot 3.5^{2}}{0.5}}=15.5 \mathrm{M} / \mathrm{c}
$$

Based on the values of the peripheral speed, it is possible to determine the diameter of the plate at various values of the rotational speed by the formula $\mathrm{d}$

$$
d=\frac{60 \mathrm{~V}}{\pi n},
$$

Consequently, the diameters of the plates for the given rotation speeds will be (table 1):
Table 1. Font styles for a reference to a journal article.

\begin{tabular}{|c|c|}
\hline Rotational speed n, $\mathbf{~ m i n}^{-\mathbf{1}}$ & Plate diameter d, m \\
\hline 540 & 0.55 \\
\hline 720 & 0.41 \\
\hline 980 & 0.30 \\
\hline
\end{tabular}

These values give reason to believe that, given the design restrictions on the width, it is most expedient to use a spreading disc with a diameter of $0.30 \mathrm{~m}$ and a rotational speed of $980 \mathrm{~min}^{-1}$.

\section{Conclusion}

It was found that for the conditions of the above design constraints it is needed to improve its design while maintaining the advantages of the experimental model of the fertilizer spreader of the Kursk State Agricultural Academy. It is permissible to use a spreading disc with a diameter of at least $300 \mathrm{~mm}$ at its rotation frequency of no more than $980 \mathrm{~min}^{-1}$.

\section{References}

1. A.A. Shwarz et al. Science in Central Russia. 6 (48), 21-29. (2020).

2. A.A. Shwarz et. al. Science in Central Russia. 1 (49), 12-22. (2021).

3. A.S. Andreev et. al. Intensive technology of cultivation of grain crops: recommendations (Minsk, Urajay. 1986).

4. I.V. Korotkov Science of the Young - the future of Russia, 4, 376-380 (2020).

5. B.P. Besedin Bulletin of the Kursk State Agricultural Academy. 7, 66-69. (2016).

6. Y.I. Yakimov, S.I. Volosnikov Tractors and agricultural machines. 12, 27-28 (1967).

7. Y. Sintsova Grach 900 - ideal mineral fertilizer spreader. Retrieved from https://selhoztehnika.net/news-777/grach-900idealnyj-razbrasyvatel-mineralnyh-udobrenij (2015).

8. T. Larchenko RAUCH spreaders in Belarus: strength-in accuracy. Retrieved from https://agronews.com/by/ru/news/agrosfera/201807-26/rauch-v-belarusi (2018).

9. E.V. Truflyak Laboratory workshop on the use of precision farming elements. (Krasnodar, KubGAU, 2018).

10. A.S. Dobyshev et. al. Bulletin of the belarusian state agricultural academy. 1, 189-192. (2010). 
11. V.E. Komaristov, N.F. Dunay Agricultural machines (Moscow, Kolos, 1984). 